\title{
Formação em Psicologia Comunitária e os seus contributos pedagógicos para a Participação Cívica
}

\section{Training in Community Psychology: pedagogical contributions towards Civic Participation}

\author{
José Ornelas ${ }^{1}$ \\ Maria Vargas-Moniz ${ }^{1}$
}

\begin{abstract}
RESUMO
Neste trabalho procura-se documentar os processos através dos quais a formação académica avançada pode contribuir para o aprofundamento da formação cívica dos estudantes e para gerar novos polos de liderança e/ou mudança social em contextos sociais diversificados. Descreve-se o percurso do ensino da Psicologia Comunitária como formação especializada no âmbito da Psicologia e como curriculum formativo autónomo, bem como o debate sobre as competências profissionais neste domínio científico-pedagógico. Apresentam-se os resultados de um questionário sobre competências sociais e profissionais em Psicologia Comunitária e da Análise SWOT ${ }^{2}$ com estudantes de Mestrado e Doutoramento que serviram de base para a avaliação da formação pela Agência de Avaliação e Acreditação do Ensino Superior (http://www.a3es.pt/pt). A análise dos resultados permite concluir que os diplomados em Psicologia Comunitária escolheram causas cívicas diversificadas nos seus percursos profissionais e que utilizam as competências formativas adquiridas no seu percurso formativo na sua prática profissional. Através da análise qualitativa dos dados recolhidos através de análise SWOT,
\end{abstract}

\section{DOI: $10.1590 / 0104-4060.36580$}

1 Instituto Superior de Ciências Psicológicas Sociais e da Vida - Instituto Universitário. Lisboa, Portugal. Rua Jardim do Tabaco, $\mathrm{n}^{\circ}$ 34. 1149-041.

2 Método desenhado para apoiar e acompanhar a formulação e planeamento estratégico. É um processo de reflexão inclusivo e interativo que facilita o planeamento orientado para: a) os pontos fortes/ capacidades ou competências; b) os pontos frágeis no grupo e/ou organização; c) as ameaças ou constrangimentos e d) as oportunidades no contexto socioeconómico mais abrangente (DYSON, 2004). 
realça-se a relevância atribuída à liberdade de escolha durante o percurso formativo e as oportunidades de contacto com realidades sociais diversificadas. A participação em contextos comunitários tem permitindo compreender melhor a complexidade da aplicação prática dos princípios, bem como do papel que pode desempenhar a investigação com base em pressupostos de relevância e utilidade para o desenvolvimento dos indivíduos, grupos e/ou comunidades.

Palavras-chave: Psicologia Comunitária; formação; Participação Cívica.

\begin{abstract}
With this paper we aim to document how advanced academic training may contribute towards the students' civic engagement, and to generate renovated social and community leadership in diverse social contexts. We described The development of teaching Community Psychology as a special domain within Psychological Sciences, and as an autonomous curriculum, including the debate about scientific and professional competencies in that scientific-pedagogical field. The results presented derive from a survey on social and professional competencies on Community Psychology to current students of the Master's and Doctoral Programs, which, together with the results of a SWOT Analysis, formed the basis for the curriculum evaluation by the Portuguese National Agency for Evaluation and Validation of Higher Education (http://www.a3es.pt/pt). The results indicate that the graduate students have chosen diversified civic causes in their professional careers, and they use the competencies acquired during their training in their professional affairs. The qualitative data of the SWOT Analysis emphasizes that the students consider relevant the freedom of choice during the training, and the opportunity of contact and participation within diverse social realities, that facilitate the understanding of the practical application of principles, as well as the role of research anchored in assumptions of relevance and utility for the development of individuals, groups or communities.
\end{abstract}

Keywords: Community Psychology; training; Civic Participation.

\title{
Introdução
}

A Psicologia Comunitária emergiu nos anos sessenta do Século XX, no âmbito dos movimentos sociais e de defesa dos direitos humanos e num período pautado por significativas transformações sociais, entre as quais a maior consciência cívica e política acerca das consequências negativas das condições 
adversas dos contextos sociais em termos de saúde física e mental. Nessa era de inovação, estudos epidemiológicos como os de Dorenwend e Dohrenwend (1969) e de Leighton, Harding, Macklin e McMillan (1963 demonstraram a relação inversa entre as condições sociais e a emergência de problemáticas psicológicas; os trabalhos de Kurt Lewin (1946) e de Burgess e Bogue (1967) enfatizaram a relevância das variáveis ambientais na compreensão do comportamento humano, realçaram a necessidade de um debate mais aprofundado na procura de respostas alternativas e inovadoras às abordagens clínicas mais tradicionais e centradas apenas nos indivíduos (ver ALBEE, 2006 ou ORNELAS, 2008).

Uma obra de referência sobre Psicologia Comunitária foi publicada em 1977, por Julian Rappaport, da Universidade de Illinois, em Chicago, nos Estados Unidos, na qual propõe um novo paradigma para a intervenção em Psicologia, focalizado na mudança social, na relevância das alterações e ajustamentos de serviços em relação aos grupos e/ou populações em situação de maior vulnerabilidade e propondo estratégias de empoderamento para facilitar a participação social ativa. Com esta abordagem, a principal preocupação e objecto de estudo dos(as) Psicólogos(as) Comunitários(as) passa a ser a compreensão e intervenção nos processos que tenham maior potencialidade de melhorar o estado psicológico das pessoas nos contextos onde habitam e circulam (LEVINE, 1981).

Com base nestas premissas, a Psicologia Comunitária tem vindo a afirmar-se como um campo específico das ciências sociais e humanas, procurando incorporar a investigação e a ação focalizando-se, por um lado, na promoção da mudança, da justiça social e, por outro, no bem-estar individual e na ligação dos indivíduos com os contextos sociais e/ou comunitários (ORNELAS, 1997; NELSON; PRILLELTENSKY, 2005).

No desenvolvimento dos Curricula de formação em Psicologia Comunitária procura-se responder a um duplo desafio de preparar os futuros profissionais com um conjunto de princípios e valores e de lhes proporcionar o contacto com métodos e técnicas de investigação e programas de intervenção coerentes e alinhados com os pressupostos veiculados teoricamente (DALTON; ELIAS; WANDERSMAN, 2001; ORNELAS, 2008).

Os curricula de formação em Psicologia Comunitária como formação avançada (programas de Mestrado e Doutoramento) têm sido também ancorados pela emergência de sociedades científicas em diversos pontos no globo, a saber a Society for Community Research and Action (www.scra27.org), que se constitui como a $27^{\mathrm{a}}$ Divisão da American Psychological Association; a European Community Psychology Association (www.ecpa-online.eu), ou a Australian Psychology Society (www.psychology.org.au), que reconhece a Psicologia Comunitária como área especializada de intervenção em Psicologia. Estes organismos estruturam também a sua investigação e intervenção em eventos 
científicos regulares e num número significativo de publicações científicas com revisão por pares e com fator de impacto. A nível mais local existem diversos organismos científicos que congregam docentes, investigadores e profissionais em diversos países, como a Società Italiana di Psicologia di Comunità (www. sipco.it); o reconhecimento da Psicologia Comunitária como especialidade na British Psychological Society (www.bps.org.uk), ou ainda a Sociedade Portuguesa de Psicologia Comunitária (www.sppc.pt), para citar apenas alguns exemplos.

Os avanços da investigação e a ação inspirados nos pressupostos da Psicologia Comunitária têm sido relevantes para a compreensão da complexidade dos processos de mudança social e comunitária e da importância da participação cívica das populações e dos profissionais como facilitadores desses processos, contribuindo para o aprofundamento e sustentabilidade dos percursos de mudança. Estes percursos podem ter lugar em diferentes níveis ecológicos de análise (BUTTERFOSS, 2007), que podem variar desde a análise e as implicações de enquadramentos legislativos ou regulamentares, à mudança de serviços e/ou programas, e ainda focalizada nos processos de empoderamento (ver ZIMMERMAN, 2000, ou MIGUEL; ORNELAS, In press) dos indivíduos, dos grupos, organizações ou das comunidades (ver MATTON, 2008 ou JORGE-MONTEIRO et al., 2014) e na investigação colaborativa (ver TRICKETT; ESPINO, 2004; SACCHETTO; VARGAS-MONIZ, 2011 ou ORNELAS; AGUIAR; SACCHETTO; JORGE-MONTEIRO, 2012).

\section{A formação em Psicologia Comunitária}

Neste âmbito apresenta-se um breve resumo do panorama da formação em Psicologia Comunitária no contexto português que, como disciplina de ensino-aprendizagem, tem surgido como tema introdutório ou de aprofundamento integrado na formação geral em Ciências Psicológicas. Em Portugal, iniciou-se em 1987, no ISPA-IU (Instituto Superior de Ciências Psicológicas, Sociais e da Vida (www.ispa.pt), com a designação de Tema Aprofundado em Psicologia Comunitária, enquadrado na formação no âmbito do $4^{\mathrm{a}}$ Ano da formação em Psicologia Clínica.

Atualmente, observa-se uma implantação significativa da disciplina no panorama da oferta formativa integrada em planos de estudos em Psicologia. Como Curricula integrais em Psicologia Comunitária existem dois em Portugal, um no ISPA-IU, com a designação Psicologia Comunitária, e outro intitulado Psicologia Comunitária e Proteção de Menores, no ISCTE-IUL (Instituto 
Superior de Ciências do Trabalho e da Empresa - Instituto Universitário de Lisboa (www.iscte-iul.pt). No entanto, em diversos estabelecimentos de Ensino Superior são lecionadas disciplinas de Psicologia Comunitária, tanto a nível de $1^{\circ}$ como de $2^{\circ}$ Ciclo de Estudos, como nas Universidades do Porto, do Minho, do Algarve, da Beira Interior, dos Açores, na Universidade Católica de Braga, no Instituto Politécnico de Bragança, entre outros, bem como em um curso de Pós-Graduação no ISMAI - Instituto Universitário da Maia (www.ismai.pt).

No âmbito do $3^{\circ}$ Ciclo de Estudos (Doutoramento), iniciou-se no ISPA-IU, integrado no Doutoramento em Psicologia, uma área de especialidade em 2007 e conta atualmente com 16 estudantes, havendo 2 já diplomados, sendo que 6 (33\%) foram, ou são ainda, Bolseiros da Fundação para a Ciência e Tecnologia.

No sentido de compreender em que medida a formação em Psicologia Comunitária tem promovido o envolvimento e a participação cívica ativa dos seus estudantes, procurou-se enquadrar esta dimensão nos objetivos gerais e específicos definidos para o Curriculum formativo, que são o aprofundamento de modelos, teorias e técnicas, e no desenvolvimento de competências coerentes com os princípios e valores em Psicologia Comunitária e estabelecer relações substantivas com a prática de intervenção e com outras áreas científicas. Pretende-se que os(as) alunos(as) sejam capazes de selecionar métodos de investigação e intervenção e tenham a capacidade de reflexão crítica acerca da aplicabilidade das teorias em Psicologia Comunitária. Procura-se a produção de inovação, resolução de problemas e análise de situações com considerações éticas. Pretende-se também que desenvolvam capacidades de comunicação e disseminação da pesquisa e adquiram autonomia e interesse pela aprendizagem ao longo da vida (ver ACEF, 2011). No que concerne aos objetivos específicos, promove-se a coerência com os valores institucionais associados ao aprofundamento do humanismo através da promoção da filosofia de empoderamento, associada à capacidade dos indivíduos e grupos de determinarem o seu percurso de desenvolvimento e o estudo sistemático de processos de renovação e recuperação de indivíduos e grupos com experiências associadas a elevados índices de vulnerabilidade e trauma (p.e. pessoas com doença mental ou sobreviventes de violência doméstica e abuso sexual).

Enfatizam-se os processos de colaboração e a construção de parcerias entre a Universidade e a comunidade que permitem a materialização de valores associados à solidariedade, pois as intervenções têm lugar junto de indivíduos ou grupos em circunstâncias de vulnerabilidade acrescida como a pobreza extrema, configurada por exemplo nas pessoas em situação de sem-abrigo.

Ao promover-se a ligação entre os modelos e as práticas específicas em contextos comunitários, procura-se que a formação dos alunos lhes proporcione capacidades para o exercício refletido e ancorado em princípios e valores de 
uma prática coerente e promotora da autodeterminação dos indivíduos, grupos e/ou comunidades.

A miríade de unidades curriculares disponíveis procura demonstrar que a investigação e a intervenção em contextos comunitários poderá ter uma orientação proactiva, isto é, procurando agir preventivamente, antecipando a emergência das problemáticas; ou uma orientação reativa, correspondendo à construção de serviços e sistemas com a capacidade de responder eficazmente a situações ou fenómenos sociais de elevada complexidade.

No âmbito da investigação, uma prioridade em Psicologia Comunitária é a do respeito pela dignidade e integridade dos participantes, reconhecendo a validade dos seus contributos e conhecimento adquirido com base na sua experiência, procurando que os trabalhos desenvolvidos se pautem por procedimentos rigorosos de consentimento informado e de garantia do anonimato das informações concedidas (ver ACEF, 2011).

A SCRA - Society for Community research and Action - tem, desde 1987, com Elias, Dalton e Godin, procurado coligir informações a nível internacional acerca dos programas de formação avançada em Psicologia Comunitária, tendo já sido publicados diversos relatórios (p.e. HAZEL, 2007; DZIADKOWICZ; JIMENEZ, 2009; JULIAN, 2006). Considerando que a identidade específica da Psicologia Comunitária como formação científica que procura ser coerente com uma prática profissional proactiva e orientada para a mudança social, Dalton e Wolfe (2012) sistematizaram as competências profissionais (18) em Psicologia Comunitária (Tabela 1), onde se enfatizam as características centrais que devem constar dos programas de Psicologia Comunitária e as competências para a prática profissional que se pretendem desenvolver. Embora este seja um documento ainda em desenvolvimento (WOLFE; CHIEN-SCOTT; JIMENEZ, 2013), considera-se ter um significativo potencial para suscitar o debate acerca da sua aplicabilidade à escala global, no sentido de criar um perfil científico e profissional e contribuir para a consolidação pedagógica e cívica desta área de estudos em Psicologia.

A partir deste racional ancorado em quatro pilares-base que abrangem o domínio e a capacidade de aplicação dos princípios, o desenvolvimento de programas, a capacidade de delinear e acompanhar percursos de mudança social e a investigação em contextos comunitários, foi possível sintetizar de forma criativa, mas simultaneamente objetiva uma estratégia para disseminar a Psicologia Comunitária como domínio cientifico-pedagógico e como prática profissional.

O perfil profissional do Psicólogo Comunitário que emerge desta reflexão teórico-prática é coerente com as questões acerca das quais se procura refletir neste artigo: em que medida a formação em Psicologia Comunitária fomenta a participação cívica no quotidiano dos seus estudantes e graduados? Procura- 
ORNELAS, J.; VARGAS-MONIZ, M. Formação em Psicologia Comunitária e os seus contributos...

\section{TABELA 1 - COMPETÊNCIAS PARA A PRÁTICA DA PSICOLOGIA COMUNITÁRIA}

\begin{tabular}{|c|c|}
\hline Competência & Definição: "A capacidade de..." \\
\hline \multicolumn{2}{|l|}{ Princípios Fundamentais } \\
\hline A perspetiva ecológica & $\begin{array}{l}\text { Articular e aplicar perspetivas ecológicas e níveis de análise na } \\
\text { prática comunitária. }\end{array}$ \\
\hline Empoderamento & $\begin{array}{l}\text { Articular e aplicar a perspetiva de empoderamento para apoiar } \\
\text { comunidades que tenham sido marginalizadas nos seus esfor- } \\
\text { ços para aceder a recursos no processo comunitário de tomada } \\
\text { de decisão. }\end{array}$ \\
\hline $\begin{array}{l}\text { Competências socioculturais e } \\
\text { trans-culturais }\end{array}$ & $\begin{array}{l}\text { Valorizar, identificar e estabelecer pontos entre várias visões do } \\
\text { mundo, das culturas e identidades. }\end{array}$ \\
\hline $\begin{array}{l}\text { Inclusão comunitária e } \\
\text { parceria }\end{array}$ & $\begin{array}{l}\text { Promover a representação genuína e o respeito de todos os } \\
\text { membros da comunidade para agir no sentido de legitimar } \\
\text { perspetivas divergentes acerca de questões sociais e } \\
\text { comunitárias. }\end{array}$ \\
\hline Prática com reflexão Ética & $\begin{array}{l}\text { Identificar questões éticas na sua prática profissional individual } \\
\text { e agir de forma coerente e responsável. }\end{array}$ \\
\hline \multicolumn{2}{|c|}{ Desenvolvimento de Programas Comunitários } \\
\hline $\begin{array}{l}\text { Desenvolvimento de } \\
\text { programas, implementação e } \\
\text { gestão }\end{array}$ & $\begin{array}{l}\text { Estabelecer parcerias com intervenientes na comunidade } \\
\text { para planear, desenvolver, implementar e promover a } \\
\text { sustentabilidade de programas em contextos comunitários. }\end{array}$ \\
\hline $\begin{array}{l}\text { Prevenção e promoção da } \\
\text { saúde }\end{array}$ & $\begin{array}{l}\text { Articular e implementar uma perspetiva de prevenção e } \\
\text { operacionalizar programas comunitários de prevenção e } \\
\text { promoção da saúde. }\end{array}$ \\
\hline $\begin{array}{l}\text { Construção da capacidade } \\
\text { comunitária e organizacional }\end{array}$ & $\begin{array}{l}\text { Envolver, energizar e mobilizar os membros da comunidade } \\
\text { e os grupos em torno de temas de relevância partilhada. } \\
\text { Identificar as capacidades individuais e os recursos sociais e } \\
\text { estruturais para aprofundar o empoderamento, o envolvimento } \\
\text { comunitário e a liderança. }\end{array}$ \\
\hline $\begin{array}{l}\text { Processos de pequenos e de } \\
\text { grandes grupos }\end{array}$ & $\begin{array}{l}\text { Intervir em processos de grupo de pequena e grande dimensão } \\
\text { de modo a facilitar a capacidade dos grupos comunitários a tra- } \\
\text { balhar produtivamente em conjunto. }\end{array}$ \\
\hline Desenvolvimento de recursos & $\begin{array}{l}\text { Identificar e integrar a utilização de recursos humanos, incluin- } \\
\text { do os bens comunitários e o capital social. }\end{array}$ \\
\hline $\begin{array}{l}\text { Consultoria e } \\
\text { desenvolvimento } \\
\text { organizacional }\end{array}$ & $\begin{array}{l}\text { Facilitar o crescimento, a consolidação e a capacidade organi- } \\
\text { zacional para alcançar os objetivos definidos. }\end{array}$ \\
\hline \multicolumn{2}{|l|}{ Mudança Social e Comunitária } \\
\hline $\begin{array}{l}\text { Colaboração e } \\
\text { Desenvolvimento de Parcerias }\end{array}$ & $\begin{array}{l}\text { Apoiar grupos com interesses comuns para que façam juntos o } \\
\text { que não podem fazer individualmente. }\end{array}$ \\
\hline $\begin{array}{l}\text { Desenvolvimento } \\
\text { Comunitário }\end{array}$ & $\begin{array}{l}\text { Apoiar a comunidade no desenvolvimento de uma visão e im- } \\
\text { plementar ações orientadas para a construção de comunidades } \\
\text { saudáveis. }\end{array}$ \\
\hline
\end{tabular}


(Conclusão)

\begin{tabular}{l|l}
\hline $\begin{array}{l}\text { Organização Comunitária e } \\
\text { Defesa Cívica }\left(^{*}\right)\end{array}$ & $\begin{array}{l}\text { Trabalhar colaborativamente com os membros da comunidade } \\
\text { para que se alcancem maiores níveis de poder e se melhorem as } \\
\text { condições que afetam a comunidade. }\end{array}$ \\
\hline $\begin{array}{l}\text { Análise de Políticas Públicas, } \\
\text { desenvolvimento e defesa } \\
\text { cívica }\end{array}$ & $\begin{array}{l}\text { Construir e promover a sustentabilidade para uma comunicação } \\
\text { eficaz e relações de trabalho com agentes políticos, com repre- } \\
\text { sentantes eleitos e líderes comunitários. }\end{array}$ \\
\hline $\begin{array}{l}\text { Educação/Formação comunitá- } \\
\text { ria, informação, disseminação } \\
\text { e sensibilização de públicos }\end{array}$ & $\begin{array}{l}\text { Comunicar informação a vários segmentos do público para for- } \\
\text { talecer competências, sensibilização ou defesa cívica. }\end{array}$ \\
\hline Investigação Comunitária & $\begin{array}{l}\text { Trabalhar com parcerias na comunidade para planear e condu- } \\
\text { zir investigação com elevados padrões de rigor na produção de } \\
\text { evidência científica, que seja contextualmente adequada. Comu- } \\
\text { nicar os resultados da investigação de forma a promover a ca- } \\
\text { pacidade da comunidade em prosseguir os objetivos definidos. }\end{array}$ \\
\hline $\begin{array}{l}\text { Investigação Comunitária } \\
\text { Participativa }\end{array}$ & $\begin{array}{l}\text { Estabelecer parcerias com líderes e membros das comunidades/ } \\
\text { contextos para promover melhorias e a responsabilidade dos } \\
\text { intervenientes e líderes, bem como das entidades financiadoras. }\end{array}$ \\
\hline Avaliação de Programas
\end{tabular}

FONTE: Traduzido e adaptado de Dalton e Wolfe (2012), com a permissão expressa dos autores.

(*) Segundo Schneider e Lester (2001) o termo Advocacy, que se traduziu para a língua portuguesa como defesa cívica, pode ter múltiplos significados e ser utilizado em muitos campos das ciências sociais e humanas, mas pode ser sintetizado como uma intervenção a favor de uma pessoa em termos da organização de serviços ou suportes, assegurando a sua acessibilidade e relevância. No que concerne os grupos e/ou comunidades, centra-se na organização de capacidades de organização, planeamento social e colaboração intersectorial orientados para a promoção da justiça social (Cf. GAMBLE, M. O.; GAMBLE, D. N., 1995). (N. dos A)

-se também compreender como é que a investigação ou a Ciência Comunitária contribuem para o aprofundamento democrático e para a promoção dos Direitos Humanos e, finalmente, uma terceira questão: como pode a Psicologia Comunitária gerar polos de liderança e mudança em domínios sociais diversificados?

Considera-se que a partir deste tipo de proposta, estaremos mais habilitados a refletir com parceiros diversificados, privilegiando os falantes de língua portuguesa acerca do papel da formação em Psicologia Comunitária como instrumento inovador para a participação cívica e comunitária.

\section{Método}

Considerou-se como pertinente para documentar esta reflexão a utilização de uma estratégia eminentemente qualitativa, com componentes quantitativos, 
mas de carácter estritamente descritivo, por permitir o estudo mais detalhado do grupo de estudo, neste caso, os estudantes e diplomados com mestrado e/ou doutoramento em Psicologia Comunitária. Segundo Patton (1990), os métodos qualitativos assumem relevância na análise das experiências educativas, por permitirem estudar os temas com maior profundidade e detalhes, obtendo-se informações que envolvem opiniões ou relatos na primeira pessoa acerca de uma experiência específica e a análise do contexto sociopolítico em estudo (ver também LAPAN; QUARTAROLI; RIEMER, 2012, p. 5): “explicar por palavras próprias as ideias acerca de um domínio ou tema". O método qualitativo e descritivo foi também pertinente por outras duas ordens de razão, a exiguidade da amostra recolhida $(\mathrm{N}=24)$ e a natureza da informação recolhida junto dos participantes (SCHENSUL, 2012), neste caso um questionário online orientado para a reflexão individual acerca do seu percurso formativo e os resultados de uma análise SWOT sobre a formação em Psicologia Comunitária. A estruturação do questionário online foi inspirada no enquadramento do referencial de competências para a prática da Psicologia Comunitária (DALTON; WOLFE, 2012), traduzida e adaptada para a língua portuguesa, e a análise SWOT sobre a formação em Psicologia Comunitária foi realizada num conjunto de sessões com alunos e alunas de Mestrado e Doutoramento procurando recolher testemunhos sobre o papel da formação no seu desenvolvimento pessoal. O método de análise SWOT pode ser, segundo David (2011), um instrumento relevante quando se pretende perscrutar opiniões acerca de um tema específico e organizar os resultados num conjunto estratégico coerente orientado para aspetos fortes/ positivos ou negativos/fracos, conjugados com fatores internos e externos de constrangimentos e/ou oportunidades, permitindo recolher ideias e analisá-las à luz de um contexto sociopolítico mais abrangente. Considerou-se pertinente a selecção deste método, por se tratar de um exercício de reflexão crítica multidimensional, procurando associar as aprendizagens académicas, as potencialidades individuais e as oportunidades para o exercício, aplicação e/ou aprofundamento das competências e capacidades profissionais.

Para além disso, os desenhos de investigação eminentemente qualitativos são pertinentes face às características específicas da população em estudo e, ainda, como estratégia para preservar os relatos destes participantes para memória futura (BERNARD; RYAN, 2010). No presente estudo, estas considerações são relevantes, dada a natureza transitória e relativamente curta da relação pedagógica e de contacto regular da Universidade com os estudantes (4 semestres letivos no Mestrado e 2 semestres letivos no Programa Doutoral, acrescidos dos anos de tutoria individual e em grupo para a realização da tese). 


\section{Resultados}

Os resultados foram estruturados de forma a proporcionar uma descrição da população em estudo e procurar responder às questões de investigação formuladas. O questionário online foi remetido a um total de 56 alunos e antigos alunos, sendo que 42 foram alunos de Mestrado e 14 de Doutoramento, sendo a taxa de resposta de $43 \%$. Deste modo e, considerando a exiguidade da amostra, apresentam-se resultados descritivos no que concerne a sua caracterização, bem como os dados referentes às competências profissionais exercidas no domínio da Psicologia Comunitária.

Do total de respostas obtidas, $(\mathrm{N}=24),[\mathrm{n}=11(45,5 \%)]$ são de participantes no programa doutoral do ISPA-IU, que correspondem a 7 na fase de construção da tese, de 3 diplomados e, do Mestrado, 14 diplomados. No que concerne ao gênero, 15 participantes (63\%) são do sexo feminino e 9 participantes, do sexo masculino (37\%). No que concerne a faixa etária, $12(50 \%)$ têm idades compreendidas entre os 20 e os 30 anos, $17 \%$ (4) entre os 31 e os 40 anos, $25 \%$ (6) entre os 41 e os 50 anos e, com mais de 51 anos, 2 participantes (8\%). Quanto ao exercício, no momento presente, de atividade profissional na área da Psicologia Comunitária $(n=22) 92 \%$ reportam afirmativamente, estando uma pessoa a trabalhar noutra área e outra em situação de desemprego.

No que concerne às competências profissionais exercidas pelos participantes, na Tabela 2 apresentam-se os resultados para cada competência profissional, constatando-se uma diversidade de competências que são cumulativamente exercidas pelos participantes. Destacam-se, nas competências específicas, a colaboração e desenvolvimento de parcerias mencionados por $23(95.8 \%)$ dos 24 participantes e a menos referenciada ( 2 participantes, $8.3 \%$ ), prevenção e promoção da saúde. De notar será também que, no domínio dos princípios fundamentais, a adoção da perspetiva ecológica e da filosofia de empoderamento se destacam, sendo mencionadas por $62,5 \%$ e $70.8 \%$ dos participantes, respetivamente.

Analisando-se as competências profissionais a partir dos domínios propostos no referencial (ver Gráfico 1), reafirma-se a distribuição diversificada e um equilíbrio geral entre a aplicação dos princípios fundamentais $(30.3 \%)$, o desenvolvimento de programas comunitários (26.6\%) e a mudança social e comunitária $(32.5 \%)$, já a investigação é proporcionalmente menos referida $(10.6 \%)$.

Para a pergunta acerca da medida em que a atividade profissional permite aplicar os conhecimentos na área da Psicologia Comunitária, constata-se um 
ORNELAS, J.; VARGAS-MONIZ, M. Formação em Psicologia Comunitária e os seus contributos...

TABELA 2 - COMPETÊNCIAS SOCIAIS E PROFISSIONAIS EXERCIDAS

\begin{tabular}{|c|c|c|c|}
\hline Domínio & Competências & $\mathbf{N}$ & $\%$ \\
\hline \multirow{4}{*}{$\begin{array}{l}\text { Princípios } \\
\text { Fundamentais }\end{array}$} & A perspetiva ecológica & 15 & 62.5 \\
\hline & Empoderamento & 17 & 70.8 \\
\hline & Competências socioculturais e transculturais & 10 & 41.6 \\
\hline & Inclusão comunitária e parceria & 15 & 62.5 \\
\hline \multirow{6}{*}{$\begin{array}{l}\text { Desenvolvimento } \\
\text { de Programas } \\
\text { Comunitários }\end{array}$} & $\begin{array}{l}\text { Desenvolvimento de programas, implementação e } \\
\text { gestão }\end{array}$ & 15 & 62.5 \\
\hline & Prevenção e promoção da saúde & 2 & 8.3 \\
\hline & $\begin{array}{l}\text { Construção da capacidade comunitária e } \\
\text { organizacional }\end{array}$ & 10 & 41.6 \\
\hline & Processos de pequenos e de grandes grupos & 14 & 58.3 \\
\hline & Desenvolvimento de recursos & 9 & 37.5 \\
\hline & Consultoria e desenvolvimento organizacional & 11 & 45.8 \\
\hline \multirow{5}{*}{$\begin{array}{l}\text { Mudança Social e } \\
\text { Comunitária }\end{array}$} & Colaboração e Desenvolvimento de Parcerias & 23 & 95.8 \\
\hline & Desenvolvimento Comunitário & 8 & 33.3 \\
\hline & Organização Comunitária e Defesa Cívica & 9 & 37.5 \\
\hline & Análise de Políticas Públicas & 11 & 45.8 \\
\hline & $\begin{array}{l}\text { Educação/ Formação comunitária, informação, } \\
\text { disseminação e sensibilização de públicos }\end{array}$ & 10 & 41.6 \\
\hline \multirow{2}{*}{$\begin{array}{l}\text { Investigação } \\
\text { Comunitária }\end{array}$} & Investigação Comunitária Participativa & 11 & 45.8 \\
\hline & Avaliação de Programas & 9 & 37.5 \\
\hline
\end{tabular}

FONTE: Questionários de Competências Sociais e Profissionais em Psicologia Comunitária, traduzido e adaptado por Ornelas e Vargas-Moniz, de Dalton e Wolfe (2012).

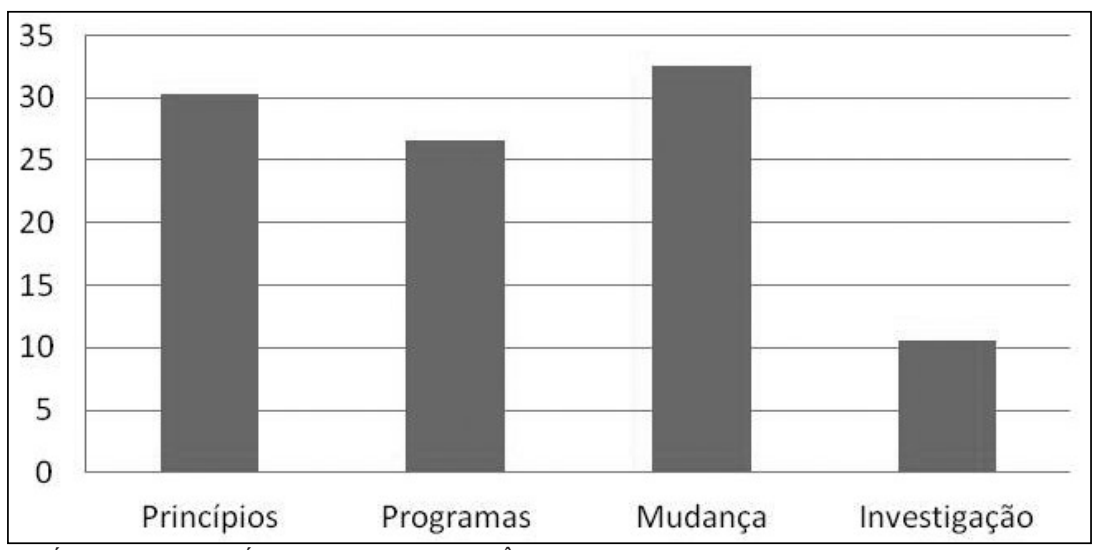

GRÁFICO 1 - DOMÍNIOS DAS COMPETÊNCIAS PROFISSIONAIS 
resultado significativamente positivo (ver Gráfico 2), relativo aos valores de 1 a 3 na escala da Likert (correspondendo a valores mais baixos) de 2 participantes, opinando os remanescentes 22 (91.6\%) que na sua atividade profissional tinham oportunidade de aplicar os conhecimentos adquiridos na área da Psicologia Comunitária, sendo que $5(20.8 \%)$ se posicionam em 4, $6(25 \%)$ em 5 e 11 (45.8\%) em 6.

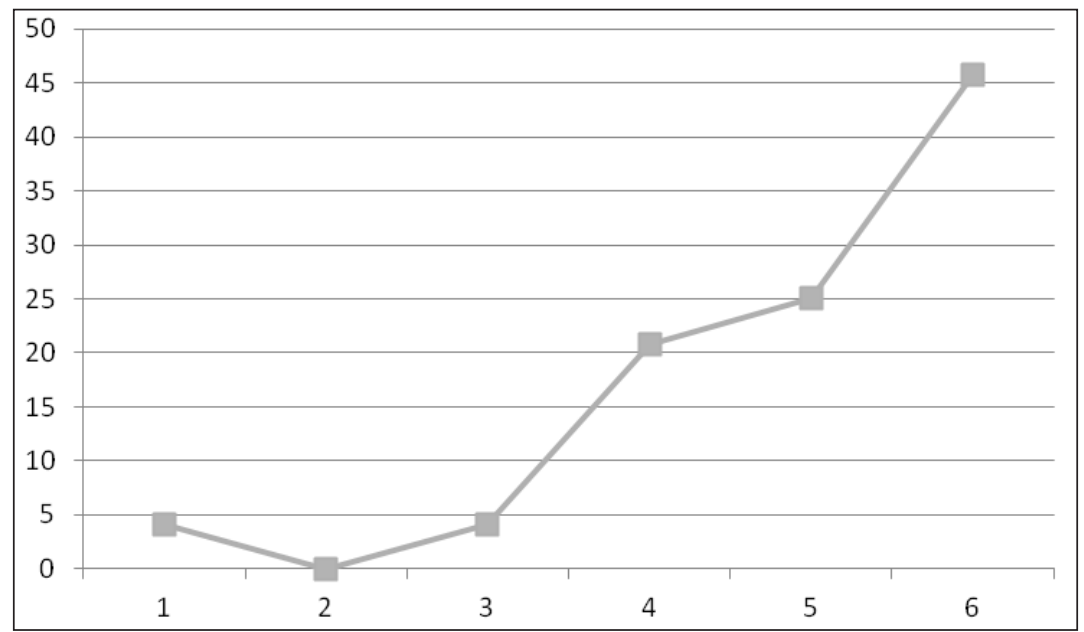

GRÁFICO 2 - APLICAÇÃO DE CONHECIMENTOS ADQUIRIDOS

Quanto à área específica de exercício de atividade profissional, constata-se um panorama diversificado abrangendo a área da saúde, incluindo a saúde mental comunitária e a prevenção da aids, o ensino e a formação de 4 participantes em ambas as áreas, envolvendo 3 participantes na área da violência contra as mulheres, 3 na área da habitação e da investigação; áreas como a avaliação de programas e o emprego de jovens apresentam um participante e, finalmente, uma pessoa trabalha noutra área profissional e outra ainda reporta uma situação de desemprego.

No que concerne os resultados da análise SWOT da apreciação dos alunos e antigos alunos sobre a formação em Psicologia Comunitária, realça-se que referem ser uma proposta científica e pedagógica com objetivos claros e diversificados, coerentes e orientados para o desenvolvimento dos indivíduos e das comunidades ["Os temas do curriculum são relevantes para o atual contexto social... desde que comecei o Mestrado sinto que aumentei a minha cultura global pela abrangência dos temas abordados." (P6, 2012); "Percebemos logo que a PC tem aplicabilidades diversificadas" (P4, 2012); ou ainda: "Senti 
sempre que teria a liberdade para escolher estudar temas que fossem do meu interesse" (P2, 2012)]. Em relação à capacidade da formação para fomentar a participação cívica no quotidiano dos seus estudantes e graduados, é fomentada liberdade individual na escolha de temas de interesse - ver, p.e. "sentimo-nos livres para pesquisar, encontrar soluções e estabelecer as ligações com outros domínios científicos" (P4, 2012). No que concerne ao corpo docente, os alunos referem: "a experiência profissional, disponibilidade e acessibilidade dos professores contribui com muitos exemplos e ajudam a motivar"; "a promoção de conferências e a variedade de opções em termos de Unidades Curriculares promovem a amplitude dos conhecimentos"; e sobre a avaliação: "trabalhos em vez de exames permitem-nos ir mais a fundo nas questões..." (P8, 2012).

No domínio da investigação, propõe-se que a Ciência Comunitária contribua para o aprofundamento democrático e para a promoção dos Direitos Humanos, pelo que se considera que se devem proporcionar oportunidades de estudo autónomo e o exercício teórico-prático de competências de investigação e intervenção que, na opinião dos participantes, se configura como a "orientação positiva e inovadora para a análise das comunidades... não como uma fonte de problemas e incapacidades, mas como um contexto de recursos e soluções" (P5, 2012). O enquadramento da Psicologia Comunitária na Unidade de Investigação em Psicologia e Saúde também proporciona um melhor enquadramento da produtividade científica também reportada pelos participantes: "os conhecimentos são apresentados de forma aprofundada e baseada em investigação realizada" (P1, 2012); "há uma maturação do estudo que é exigente, mas permite aprender mais." (P4, 2012); ou ainda: "termos professores de outras Universidades nacionais e estrangeiras aumenta a nossa compreensão da abrangência e do potencial da Psicologia Comunitária" (P6, 2012).

No que se refere ao desafio de gerar polos de liderança e mudança em domínios sociais diversificados, importa realçar que os domínios temáticos de estudo que, em muitas circunstâncias, englobam componentes de ação, envolvem temas como a desinstitucionalização (VARGAS-MONIZ; BRUGES-COSTA; ORNELAS, 2013), a integração comunitária e o empoderamento de pessoas com experiência de doença mental (ORNELAS et al., 2012a e 2012b), pessoas em situação de sem-abrigo, sobreviventes de abuso sexual (MARIA; ORNELAS, 2012), violação ou violência doméstica (CARDOSO; ORNELAS, 2013), migrantes indocumentados, bem como a participação cívica e comunitária dos jovens, neste último campo realçando-se o prémio de mérito recebido por tese de mestrado (CABRAL; ORNELAS, 2012), demonstrando nestes domínios de investigação e intervenção interesse a nível nacional e internacional pela sua aceitabilidade em eventos científicos nacionais e internacionais e pela produção científica crescente publicada, por um lado, e pelas organizações que emergiram 
a partir destes temas e com quem se mantêm parcerias universidade/comunidade com actividade consistente e continuada de colaboração.

No que diz respeito às propostas de acção para melhorar o panorama da Psicologia Comunitária a nível nacional e internacional, coligiram-se as opiniões dos participantes na Tabela 3, que está estruturada a partir de três eixos-base: a) divulgação e disseminação; b) certificação e reconhecimento profissional e; c) ideias e inovação, sendo relevante notar que estes são os contributos diretos referidos pelos participantes, a partir do questionário online.

TABELA 3 - PROPOSTAS DE MELHORIA NO PANORAMA DA PSICOLOGIA COMUNITÁRIA

\begin{tabular}{l|l}
\hline \multirow{2}{*}{$\begin{array}{l}\text { Divulgação e } \\
\text { Disseminação }\end{array}$} & $\begin{array}{l}\text { Aumento das Publicações em Língua Portuguesa e a nível internacional } \\
\text { Mais oportunidades de apresentação e disseminação da pesquisa e da evi- } \\
\text { dência científica } \\
\text { Maior número de acções de formação fora do contexto académico; } \\
\text { Maior visibilidade nos media. }\end{array}$ \\
\hline $\begin{array}{l}\text { Certificação e } \\
\text { Reconhecimento } \\
\text { Profissional }\end{array}$ & $\begin{array}{l}\text { Reconhecimento da Psicologia Comunitária como área específica das Ciên- } \\
\text { cias Psicológicas na Ordem dos Psicólogos Portugueses; } \\
\text { Consolidação das redes de ligação entre a academia e a prática profissional. }\end{array}$ \\
\hline & $\begin{array}{l}\text { Construção de comunidades saudáveis e com maior índice de desenvolvi- } \\
\text { mento humano; } \\
\text { Ideias e Inovação } \\
\text { Perceber como a Psicologia Comunitária contribui para a melhoria da so- } \\
\text { ciedade; } \\
\text { Sustentabilidade dos programas comunitários; } \\
\text { Fomentar a integração comunitária; } \\
\text { Maior influência nas políticas públicas. }\end{array}$ \\
\hline
\end{tabular}

FONTE: Questionário de Competências Sociais e Profissionais em Psicologia Comunitária, traduzido e adaptado por Ornelas e Vargas-Moniz, de Dalton e Wolfe (2012).

Considera-se assim que este conjunto de resultados proporciona um panorama geral sobre o que tem sido realizado, mas conduz à necessidade de um renovado e mais aprofundado esforço de aprofundamento e internacionalização das práticas pedagógicas em Psicologia Comunitária.

\section{Discussão e conclusões}

Retomando a primeira das questões de investigação formuladas acerca da medida em que a formação em Psicologia Comunitária proporciona opor- 
tunidades para a participação cívica no quotidiano, constata-se que a partir do perfil descritivo das competências profissionais exercidas, quando combinado com as áreas profissionais de envolvimento e ainda com a produção em termos de investigação, que há uma diversidade de causas às quais os participantes se dedicam. Valoriza-se a liberdade e a oportunidade de escolha no percurso de ensino/aprendizagem e definição dos interesses individuais ou escolhas formuladas no decurso do percurso formativo e o resultado prático desta forma de pedagogia tem como resultado prático um envolvimento ativo em causas cívicas socialmente relevantes (ver p.e. ORNELAS; VARGAS-MONIZ; DUARTE, 2010 ou NELSON; KLOSS; ORNELAS (In Press) na área da saúde mental comunitária, ou (CARDOSO; ORNELAS, 2013) na área da violência contra as mulheres, ou ainda (MARIA; ORNELAS, 2012) na prevenção do abuso sexual de crianças, sendo este resultado consistente com a reflexão de Wolfe (2014) acerca da utilidade prática da utilização intercorrente das diversas competências e a sua relevância para a afirmação da psicologia comunitária a nível local e em simultâneo na influência das políticas públicas, utilizando como exemplo o trabalho desenvolvido para reduzir as disparidades no acesso à saúde.

No que concerne a segunda questão de investigação acerca de como a Ciência Comunitária pode contribuir para o aprofundamento democrático e para a promoção dos Direitos Humanos, de acordo com Connell et al. (2013), ou Münger (2012), sobre a especificidade dos Psicólogos Comunitários como peritos cívicos, pelo seu entendimento da relevância da colaboração entre todos os intervenientes no contexto comunitário e de que a eficácia e os resultados da colaboração requerem processos planeados e deliberados, realça-se que nos resultados obtidos a competência profissional mais referenciada pelos participantes foi o trabalho em parceria e, no domínio da investigação, os estudos de Vargas-Moniz e Morgado (2012), ou Ornelas e Vargas-Moniz (2011) são também um exemplo dessa relevância, com um estudo a nível nacional da eficácia e dos resultados em Comissões de Proteção de Crianças e Jovens e, ainda, Ornelas et al. (2013), que reporta um estudo colaborativo com líderes comunitários. Quanto à capacidade de gerar polos de liderança e mudança em domínios sociais diversificados que se constituiu como a terceira questão para esta reflexão, apesar dos resultados já alcançados com a criação de diversas organizações nas áreas sociais já mencionadas como a Associação para o Estudo e Integração Psicossocial para a área da Saúde Mental, a Associação de Mulheres contra a Violência, ou a Associação Portuguesa para o Estudo e Prevenção do Abuso Sexual de Crianças, há ainda um caminho a percorrer nos termos do que os participantes propõem e que se apresenta na Tabela 3 , a respeito da sistematização de contributos para a construção e sustentabilidade de projetos e programas de comunitários, de promoção da integração comunitária e de maior influência nas 
políticas públicas. Conclui-se assim que a liberdade de pesquisa e a oportunidade de aprofundamento de estudo e de contacto com causas cívicas relevantes pode constituir-se como um valor para desenvolver ciência comunitária e lideranças renovadas em contextos e/ou situações diversificadas.

\section{REFERÊNCIAS}

ACEF/1112/12662 - Guião para a auto-avaliação, 2011. Disponível em: $<$ http://www. a3es.pt/si/iportal.php/process_form/print?processId=8549... > . Acesso em: 08/05/2014.

ALBEE, G. Historical overview of primary prevention of psychopathology: address to the 3rd World Conference on the Promotion of Mental Health and Prevention of Mental and Behavioral Disorders. September 15-17, 2004. The Journal of Primary Prevention, v. 27 , n. 5 , p. $449-456,2006$.

BERNARD, H. R.; RYAN, G. W. Analyzing qualitative data: systematic approaches. Thousand Oaks, CA: Sage Publications, 2010.

BURGESS, E. W.; BOGUE, D. J. Urban Sociology. Chicago: University of Chicago, 1967.

BUTTERFOSS, F. D. Coalitions and Partnerships in Community Health. San Francisco: Jossey-Bass, 2007.

CABRAL, C.; ORNELAS, J. Empowerment dos jovens: adaptação e validação da escala de controlo sociopolítico para a população portuguesa. Tese (Mestrado em Psicologia Comunitária) - Lisboa: ISPA-IU, 2012.

CARDOSO, R.; ORNELAS, J. The changes on intimate partner violence against women in the health system in Portugal. Health, v. 5, n. 7A4, p. 65-70, 2013. doi:10.4236/ health.2013.57A4010.

CONNELL, C. M.; LEWIS, R. K.; COOK, J.; MEISSEN, G.; WOLF, T.; JOHNSONHAKIM, S.; ANGLIN, A.; FORDEN, C.; GU, B.; GUTIERREX, R.; HOSTETLER, A.; PETERSON, J.; SASAO, T.; TAYLOR, S. Special report: graduate training in community psychology practice competencies: responses to the 2012 Survey of Graduate Programs in Community Psychology. The Community Psychologist, v. 46, n. 4, p. 5-8, 2013.

DALTON, J.; JULIAN, D. (Eds.). Special section: Infusing community practice into graduate training: Broadening the conversation. The Community Psychologist, v. 42, n. 4, p. 9-23, 2009.

DALTON, J. H.; ELIAS, M. J., WANDERSMAN, A. Community psychology: Linking individuals and communities. Belmont, CA: Wadsworth, 2001. 
DALTON, J.; WOLFE, S. Competencies for community psychology practice: Society for Community Research and Action draft, August 15, 2012. The Community Psychologist, v. 45, n. 4, p. 7-14, 2012.

DALTON, J.; ELIAS, M.; JULIAN, D. (Eds.). Defining competencies for practicing community psychology. The Community Psychologist, v. 40, n. 2, p. 81-105, 2007.

DAVID, F. R. Strategic Management: Concepts and cases. Boston: Prentice Hall, 2011.

DOHRENWEND, B. P.; DOHRENDWEND, B. S. Social Status and Psychological Disorder. New York: Wiley, 1969.

DYSON, R. G. Strategic development and SWOT analysis at the University of Warwick. Journal of Operational Research, v. 152, p. 631-640, 2004. Disponível em: <http:// dx.doi.org/10.1016/S0377-2217(03)00062-6>. Acesso em: 18/05/2014.

DZIADKOWICZ, O.; JIMENEZ, T. Educating community psychologists for community practice: A survey of graduate training programs. The Community Psychologist, v. 42, n. 4, p. 10-17, 2009.

ELIAS, M. J.; DALTON, J. H.; GODIN, S. A survey of graduate education in community psychology. The Community Psychologist, v. 20, n. 3, p. 10-33, 1987.

GAMBLE, M. O.; GAMBLE, D. N. Community practice models in social work encyclopedia. 19th Edition. Washington, DC: NASW Press, 1995. p. 577-593.

HAZEL, K. Infusing practice into community psychology graduate education. The Community Psychologist, v. 40, n. 2, p. 81-88, 2007.

JORGE-MONTEIRO, M. F.; AGUIAR, R.; SACCHETTO, B.; VARGAS-MONIZ, M.; ORNELAS, J. What transformation? A qualitative study on empowering settings and community mental health organizations. Special Issue in Transformative Change in Community Mental Health. Global Journal of Community Psychology Practice, v. 5, n. 1, p. 1-13. Disponível em: <http://www. gjcpp.org/>. Acesso em: 10/05/2014.

JULIAN, D. A. Defining community psychology practice: Meeting the needs and realizing the dreams of the community. The Community Psychologist, v. 39, n. 4, p. 66-69, 2006.

LAPAN, S. D.; QUARTAROLI, M. T.; RIEMER, F. J. (Eds.). Qualitative research: an introduction to methods and designs. San Francisco, CA: Jossey-Bass, 2012.

LEIGHTON, D. C.; HARDFING, J. S.; MACKLING, D. B.; MACMILLAN, A. M.; LEIGHTON, A. H. The character of danger: Psychiatric symptoms in selected communities ("The Stirling County Study of Psychiatric disorder and sociocultural environment", Vol. III). New York: Basic Book, Inc, 1963.

LEVINE, L. The history and politics of community mental health. New York: Oxford University Press, 1981.

LEWIN, K. Action research and minority problems. Journal of Social Issues, v. 2, n. 4, p. 34-46, 1946. 
MARIA, S.; ORNELAS, J. Abusos sexuais de crianças: Mudanças resultantes de uma intervenção preventiva. Lisboa: ISPA - IU, 2012. Disponível em: <http:/hdl.handle. net/10400.12/1768>. Acesso em: 08/05/2014.

MATON, K. I. Empowering community settings: Agents of individual development, community betterment, and positive social change. American Journal of Community Psychology, v. 41, n. 1-2, p. 4-21, 2008. doi: 10.1007/s10464-007-9148-6.

MIGUEL, M.; ORNELAS, J. Defining Psychological Empowerment Construct: Analysis of three empowerment scales. Journal of Community Psychology. In Press.

MONIZ, M. J.; BRUGES-COSTA, D.; ORNELAS, J. Deinstitutionalization of seniors with mental illness: The implementation of a model of transition and integration in the community. Revista Portuguesa de Enfermagem de Saúde Mental, Porto, n. 10, dez. 2013. Disponível em: <http://www.scielo.gpeari.mctes.pt/scielo.php?script=sci arttext\&pid=S1647-21602013000200002\&lng= pt\&nrm=iso $>$. Acesso em: 22/04/2014.

MÜNGER, F. Contributing to environmental sustainability: Community Psychology's prospective role towards environmental Sustainability. The Community Psychologist, v. 45, n. 4, p. 17-20, 2012.

NELSON, G.; PRILLELTENSKY, I. Community psychology: in pursuit of liberation and well-being. New York: Palgrave Macmillan, 2005.

NELSON, G.; KLOOS, B.; ORNELAS, J. (Eds.). Community psychology and community mental health: Towards transformative change. New York: Oxford University Press. In press.

ORNELAS, J. Psicologia comunitária: Origens, fundamentos e áreas de intervenção. Análise Psicológica, 3(XV), p. 375-388, 1997.

ORNELAS, J. O Modelo Comunitário de Intervenção em Saúde Mental na Era Pós-Hospitalar. In: ORNELAS, J.; MONTEIRO, F. J.; VARGAS-MONIZ, M. J.; DUARTE, T. (Coord.). Empowerment e participação das pessoas com doença mental e seus familiares. Lisboa: AEIPS Edições, 2005. p. 11-17.

ORNELAS, J. Psicologia Comunitária. Lisboa: Fim de Século, 2008.

ORNELAS, J.; AGUIAR, R.; JORGE-MONTEIRO, M. F. Fostering capability and social integration in mental health community based organizations. Community Development Journal, Oxford Jounals, Lisbon Papers, 2012a.

ORNELAS, J.; AGUIAR, R.; SACCHETTO, B.; JORGE-MONTEIRO, M. F. Community-based participatory research: A collaborative study to measure capabilities towards recovery in mental health community organizations. Psychology, Community \& Health, v. 1, n. 1, p. 3-18, 2012b. Doi: org/10.5964/pch.v1i1.5.

ORNELAS, J.; VARGAS-MONIZ, M.; DUARTE, T. Community Psychology and Social Change: A Story from the field of Mental Health in Portugal. Global Journal of Com- 
munity Psychology Practice, v. 1, n. 1, p. 21-31, 2010. Disponível em: <http://www. gjcpp.org >. Acesso em: 09/05/2014.

ORNELAS, J.; DUARTE, T.; SEIXAS, T.; JERÓNIMO, J.; MARTINS, A.; MATOS, C.; NUNES, D.; VARELAS, F.; COSTA, L. Liderança Comunitária: Estudo Colaborativo com Dirigentes Associativos. Lisboa: CPCCRD, 2013.

ORNELAS, J.; VARGAS-MONIZ, M. J. Parcerias Comunitárias. Lisboa: ISPA - IU Edições, 2011.

PATTON, M. Q. Qualitative Evaluation and Research Methods. Thousand Oaks: CA, US: Sage Publications, 1990.

RAPPAPORT, J. Community Psychology: Values, research and action. New York: Holt, Reyhart \& Winston, 1977.

SACCHETTO, B.; VARGAS-MONIZ, M. Capabilities Theory in Practice: collaborative research with people with an experience of mental illness. Proceedings of the 7th European Congress of Community Psychology: Common Values, Diverse Practices, Paris, 2011, p. 239-242. Disponível em: <http://www. psychologie-communautaire.fr/ cmsmadesimple/uploads/Proceedings_def.pdf $>$. Acesso em: 08/05/2014.

SCHEIDER, R. L.; LESTER, L. Social Work Advocacy: A new framework for action. Belmont, CA: Wadsworth/Thomson Learning, 2011.

SCHENSUL, J. J. Methodology, methods and tools in qualitative research. In: LAPAN, S. D.; QUARTAROLI, M. T.; RIEMER, F. J. (Eds.). Qualitative research: an introduction to methods and designs. San Francisco, CA: Jossey-Bass, Thousand Oaks, CA, US: Sage Publications, Inc, 2012.

TRICKETT, E. J.; ESPINO, L. R. Collaboration and social inquiry: Multiple meaning of construct and its role in creating useful and valid knowledge. American Journal of Community Psychology, v. 34, n. 1/2, p. 1-69, 2004.

VARGAS-MONIZ, M. J.; BRUGES-COSTA, D.; ORNELAS, J. Desinstitucionalização de seniores com doença mental: A implementação de um modelo de transição e integração na comunidade. Revista Portuguesa de Enfermagem em Saúde Mental, 2013.

VARGAS-MONIZ, M. J.; MORGADO, J. Eficácia percecionada e resultados de parcerias comunitárias. Lisboa: ISPA - IU, 2012. Disponível em: $<$ http://hdl.handle. net/10400.12/1769>. Acesso em: 08/05/2014.

WOLFE, S. The application of community psychology practice competencies to reduce health disparities. American Journal of Community Psychology, v. 53, p. 231-234, 2014. DOI 10.1007/s10464-013-9622-2.

WOLFE, S. M.; CHIEN-SCOTT, V.; JIMENEZ, T. R. Community Psychology Practice Competencies: A Global Perspective. Global Journal of Community Psychology Practice, v. 4, n. 4, p. 1-9, 2013. Disponível em: <http://www.gjcpp.org/>. Acesso em: 02/05/2014. 
ZIMMERMAN, M. A. Empowerment theory: psychological, organizational and community levels of analysis. In: RAPPAPORT, J.; SEIDMAN, E. (Eds.). Handbook Of Community Psychology. New York: Kluwer Academic/ Plenum, 2000. p. 43-64.

Texto recebido em 20 de maio de 2014. Texto aprovado em $1^{\circ}$ de julho de 2014. 